\title{
FATORES QUE PREDISPÕEM A EQUIPE DE ENFERMAGEM ÀS LESÕES OSTEOMUSCULARES NO EXERCÍCIO DAS ATIVIDADES LABORAIS
}

\begin{abstract}
Dênis Derly Damasceno
Enfermeiro graduado pela EFOA/CEUFE. Doutor em Fisiologia e Farmacologia pelo ICB/UFMG, professor de Ensino Básico, Técnico e Tecnológico no Instituto Federal Sudeste de Minas - Campus Barbacena. E-mail. denis.damasceno@ifsudestemg.edu.br.

Adriana Aparecida de Almeida Santos

Aluna do curso Técnico em Enfermagem do Instituto Federal Sudeste de Minas - Campus

Barbacena. E-mail. dricasantos12@yahoo.com.br

Ângela de Fátima Rocha

Aluna do curso Técnico em Enfermagem do Instituto Federal Sudeste de Minas - Campus

Barbacena. E-mail. angel_rochabq@ @otmail.com

Daniela Dias Rocha

Aluna do curso Técnico em Enfermagem do Instituto Federal Sudeste de Minas - Campus

Barbacena. E-mail. dany-rocha13@ hotmail.com

\section{RESUMO}

A literatura indica que a equipe de enfermagem apresenta elevada ocorrência de lesões osteomusculares que se desenvolvem gradualmente e acarretam incapacidades funcionais, sendo que estas lesões geram absenteísmo e afastamentos remunerados temporários ou permanentes. Portanto, com a intenção de prover um instrumento de suporte para melhorar as condições laborais dos trabalhadores de Enfermagem em hospitais o presente estudo teve por objetivo descrever os fatores causais de lesões musculoesqueléticas, relacionadas à má postura, no desempenho das atividades de enfermagem; procurando incentivar o desenvolvimento de uma consciência crítica em relação aos efeitos do ambiente dentro de um enfoque ergonômico.
\end{abstract}

PALAVRAS-CHAVE: trabalhador de enfermagem, condição de trabalho, lesões musculoesqueléticas.

\section{RISK FACTORS THAT CONTRIBUTE TO THE DEVELOPMENT MUSCULOSKELETAL DISORDERS IN THE OCCUPATIONAL ACTIVITIES OF THE NURSING STAFF}

\begin{abstract}
A high rate of musculoskeletal disorders in members of the nursing team is observed in the literature, these disorders develop gradually and cause functional incapacity generating absenteeism, leading to temporary or permanent paid leave. Thus, with the intention to provide an instrument with support to improve the labor conditions of the workers of Nursing in hospitals the objective of this study was to describe the main causes of musculoskeletal disorders in relation to the effects of incorrect positions, in the performance of the activities of nursing; looking for to inside stimulate the development of a critical conscience in relation to the effect of the environment of an ergonomic approach.
\end{abstract}

KEY WORDS: nursing personnel, work conditions, musculoskeletal disorders 


\section{FATORES QUE PREDISPÕEM A EQUIPE DE ENFERMAGEM ÀS LESÕES OSTEOMUSCULARES NO EXERCÍCIO DAS ATIVIDADES LABORAIS.}

\section{INTRODUÇÃO}

As lesões osteomusculares são um importante problema de Saúde Pública e um dos mais graves relacionados à saúde do trabalhador (ALEXANDRE; ANGERAMI e MOREIRA FILHO, 1996; COURY; MOREIRA e DIAS, 2009). Estas lesões acometem trabalhadores em países desenvolvidos e subdesenvolvidos levando-os a diferentes graus de incapacidade funcional. Em todo o mundo, geram aumento de absenteísmo e de afastamentos temporários ou permanentes produzindo custos expressivos em tratamento e indenizações (BRASIL, 2003).

Dentre as profissões da área da saúde, a enfermagem, em particular, tem sido especialmente afetada pelas lesões osteomusculares. Pesquisas realizadas em vários países exibem prevalências superiores a $80 \%$. No Brasil a prevalência varia de $43 \%$ a 93\% (MAGNANO et al., 2007). Comélio e Alexandre (2005) e Pereira Filho; Oliveira e Cardoso (2006) mencionam que a equipe de enfermagem apresenta uma elevada ocorrência de lesões osteomusculares, principalmente as algias vertebrais, causadas por diversos fatores interrelacionados como o tipo de atividade ocupacional associado às condições ergonômicas inadequadas. Para Coury; Moreira e Dias (2009) as disfunções músculo-esqueléticas presentes no ambiente ocupacional estão associadas a fatores de risco individuais e biomecânicos. Estas disfunções se desenvolvem gradualmente, apresentam um curso crônico e, muitas vezes, permanecem sem tratamento.

Os principais fatores de risco relacionados aos distúrbios músculo-esqueléticos são a organização do trabalho (aumento da jornada de trabalho, horas extras excessivas, ritmo acelerado, déficit de trabalhadores); os fatores ambientais (espaço restrito, mobiliários inadequados, iluminação insuficiente) e as possíveis sobrecargas de segmentos corporais ocasionadas por trabalho repetitivo, movimentação e transferência de pacientes, falta de treinamento para o uso de equipamentos, técnicas e práticas de levantamento impróprias, manutenção de posturas inadequadas e estáticas, movimentos frequentes de flexão e torção da coluna vertebral e esforço físico (RADOVANOVIC e ALEXANDRE, 2002; MAGNAGO et al., 2007).

Os procedimentos que envolvem a movimentação e o transporte de pacientes são considerados os mais prejudiciais à saúde dos profissionais de enfermagem (GALLASCH e ALEXANDRE, 2003). Estas atividades são fisicamente desgastantes, possuem diferentes níveis de sobrecarga e podem tornar-se perigosas devido à má postura corporal adotada pelo profissional. A falta de manutenção de equipamentos e a utilização de mobiliários improvisados e inadequados, também, contribuem para tornar mais árduo o trabalho da enfermagem (MARZIALIE e CARVALHO, 1998; MARZIALE e ROBAZZI, 2000; GURGUERIA, ALEXANDRE e CORREA FILHO, 2003), que em grande maioria queixamse de já terem sentido dores nas costas após suas atividades de labor. Esse fato auxilia a supor que as atribuições profissionais e o ambiente de trabalho colaboram para esta queixa de dor (ALEXANDRE; ANGERAMI; MOREIRA FILHO, 1996).

$\mathrm{Na}$ literatura pesquisada foram encontrados artigos que investigaram os fatores pessoais e do ambiente de trabalho que se relacionam com a ocorrência de lesões osteomusculares na equipe de enfermagem. Nos artigos avaliados, os questionamentos voltam-se para aspectos amplos como a organização do trabalho, estrutura ambiental, conhecimento ergonômico e características antropométricas e psicológicas dos profissionais. Portanto, baseando-se na 
atualidade do tema e na falta de adequação ergonômica ainda presente nos hospitais; propusemo-nos a elaborar este estudo com o objetivo de descrever os fatores causais de lesões musculoesqueléticas, relacionadas à má postura, no desempenho das atividades de enfermagem; procurando incentivar o desenvolvimento de uma consciência crítica em relação aos efeitos do ambiente dentro de um enfoque ergonômico.

\section{METODOLOGIA}

O presente estudo trata-se de uma pesquisa exploratória, descritiva por meio de levantamento bibliográfico em base de dados. Realizou-se uma revisão bibliográfica a partir de artigos obtidos por meio de: Biblioteca Científica Eletrônica em Linha (Scientific Eletronic Library Online - SCIELO), Biblioteca Virtual de Saúde (BVS) e LILACS e periódicos sobre segurança no trabalho. Foram utilizados como descritores os seguintes termos: DISFUNÇÕES/DISTÚRBIOS MÚSCULO-ESQUELÉTICOS e ENFERMAGEM, LESÕES OSTEOMUSCULARES e ENFERMAGEM, ERGONOMIA e POSTURA, ENFERMAGEM e ERGONOMIA, ERGONOMIA NO TRABALHO.

Para a seleção dos artigos não foi avaliado a data de publicação, mas sim considerados os seguintes critérios de inclusão: estar escrito em língua portuguesa e abordar temas relacionados à importância da prevenção de agravos ao sistema músculo-esquelético ocasionados pela má postura corporal adotada pelo profissional de enfermagem durante o período laboral.

Em um primeiro momento, os artigos foram selecionados por meio do título, em seguida, pelos resumos. Foram avaliados previamente 30 textos de acordo com os critérios acima mencionados; após a leitura, foram referenciados 21 trabalhos que integram este estudo.

\section{REFERENCIAL TEÓRICO}

Os hospitais estão associados à prestação de serviços à saúde, visando o tratamento, cura ou reabilitação dos clientes por eles assistidos. No entanto, também são responsáveis pela ocorrência de uma série de riscos à saúde dos seus funcionários (GURGUERIA, ALEXANDRE e CORREA FILHO, 2003; PEREIRA FILHO; OLIVEIRA e CARDOSO, 2006). O local de trabalho, sob condições físicas, mecânicas e psíquicas adversas, é considerado como um dos principais fatores de risco para o desenvolvimento de alterações no sistema musculoesquelético. A exposição contínua e prolongada aos fatores de risco no local de trabalho favorece o surgimento das doenças ocupacionais (MAGNAGO et al., 2010).

As condições de trabalho oferecidas pelos hospitais, a crise econômica advinda da globalização, as dificuldades do setor saúde, a carência de recursos humanos e materiais e a constante preocupação com o processo de atualização, objetivando acompanhar os avanços técnicos e científicos, são fatores que contextualizam a situação dos profissionais da saúde em vários países (DEL VALLE ROYAS e MARZIALE, 2001). As condições de trabalho são intrínsecas à estrutura física e ao arcabouço organizacional da instituição e, dependendo do local de atuação e da função exercida, o trabalhador se torna, cada vez mais, predisposto aos riscos ocupacionais (MARZIALE e ROBAZZI, 2000; PEREIRA FILHO; OLIVEIRA e CARDOSO, 2006).

O trabalho de enfermagem caracteriza-se por uma série de atividades e tarefas descontínuas, que envolvem múltiplos graus de responsabilidade e complexidade segundo a relação e o tipo de função exercida (MARZIALE e ROBAZZI, 2000; DEL VALLE ROYAS e MARZIALE, 
2001). O planejamento e a programação da assistência ao paciente englobam desde as tarefas consideradas extremamente simples até as mais complexas. Portanto, são exigidos diferentes níveis de conhecimentos e habilidades para a realização das atividades laborais pela equipe de enfermagem (PEREIRA FILHO; OLIVEIRA e CARDOSO, 2006).

O absenteísmo enfrentado no serviço de enfermagem demanda uma avaliação ergonômica da unidade de trabalho, pois a ausência no trabalho pode ser considerada como indicador de inadaptação do trabalhador ao trabalho (MARZIALE e CARVALHO, 1998). Queixas relacionadas à saúde, como indisposição, insônia e fadiga decorrente das atribuições desencadeiam sobrecarga da equipe de trabalho e desfalque na escala diária afetando, desta forma, a qualidade da prestação da assistência (PEREIRA FILHO; OLIVEIRA e CARDOSO, 2006).

Um fator importante para a adaptação entre o trabalho e o homem é a formação profissional (capacitação) e o treinamento, aprimorados pela Educação Permanente. As atividades executadas pelos trabalhadores de enfermagem apresentam situações que exigem tomadas de decisões e organização de tarefas originando cargas as quais podem ser reduzidas, através de adequado preparo profissional. O treinamento constitui uma atividade organizada e programada para melhorar as habilidades de uma pessoa, ou seja, para aumentar a velocidade e a qualidade psicomotora dos movimentos necessários para se executar uma tarefa (DEL VALLE ROYAS e MARZIALE, 2001).

A falta de orientação e treinamento, assim como a jornada dupla ou trabalho sem pausas, os movimentos repetitivos e o uso de equipamentos não adequados e não ergonômicos contribuem para o agravamento de problemas osteomusculares (REIMBERG, 2006). Características sócio-demográficas (ser mulher; extremos de idade; filhos pequenos; baixa escolaridade; obesidade; tabagismo) e laborais (ser enfermeiro, técnico ou auxiliar de enfermagem; trabalho noturno; alta demanda física no trabalho) estão associadas a aumento das lesões osteomusculares e consequentemente com a dor em várias regiões (MARZIALE e CARVALHO, 1998; MAGNAGO et al., 2010).

\section{MOVIMENTAÇÃO E TRANSFERÊNCIA DE PACIENTES}

Os procedimentos que envolvem a movimentação e o transporte de pacientes são os mais realizados em ambiente hospitalar e os risco relacionados a este procedimento são elevados, sendo, considerados estes procedimentos os mais prejudiciais à saúde dos profissionais de enfermagem (GALLASCH e ALEXANDRE, 2003). As atividades de banho de chuveiro com transporte através de cadeira de banho, mudança de decúbito e arrumação de cama com paciente são fisicamente desgastantes, possuem diferentes níveis de sobrecarga e podem tornar-se perigosas devido à má postura corporal adotada pelo profissional. A falta de manutenção de equipamentos e a utilização de mobiliários improvisados e inadequados, também, contribuem para tornar mais árduo o trabalho da enfermagem (COMÉLIO e ALEXANDRE, 2005).

Durante a realização da transferência do paciente, os pesos levantados pelos trabalhadores igualam-se ou excedem as recomendações. Além disso, são realizados sob condições desfavoráveis, com número reduzido de pessoas, equipamentos inadequados e sem manutenção (ALEXANDRE, 1998). Muitos trabalhadores de enfermagem têm consciência do esforço e desgaste físico necessário para movimentar ou transportar um paciente e, também, de que se encontram despreparados em relação ao uso de técnicas posturais corretas (ZANON e MARZIALE, 2000). 
Quando se planeja a movimentação/transporte é imprescindível fazer uma avaliação completa do paciente (idade, peso, nível de consciência, capacidade de movimentação, presença de cateteres, etc.) e determinar um líder para que ocorra sincronia entre as pessoas que executarão a atividade, pois a não-sincronização ocasiona desconforto à enfermagem e ao paciente. É importante o planejamento cuidadoso da transferência, solicitando a ajuda do cliente sempre que possível, pois isto contribui para sua reabilitação e conforto, além de reduzir o esforço da equipe (ALEXANDRE e ROGANTE, 2000; ROSSI; ROCHA e ALEXANDRE, 2001).

\section{ESPAÇO FÍSICO}

O espaço físico restrito limita os movimentos interferindo na movimentação, transporte e manipulação dos pacientes. A disposição das camas no quarto, a inexistência de distâncias adequadas entre os leitos e o excesso de mobiliário contribuem para dificultar os procedimentos (ROSSI; ROCHA e ALEXANDRE, 2001; PEREIRA FILHO; OLIVEIRA e CARDOSO, 2006).

De acordo com Alexandre et al. (1992); Alexandre e Rogante (2000) e Pereira Filho; Oliveira e Cardoso (2006) os principais problemas do espaço físico são: camas posicionadas próximas as paredes laterais (disposição inadequada) e excesso de mobiliário o que torna necessário a movimentação destes equipamentos durante os cuidados de enfermagem; banheiros com espaço físico reduzido o que dificulta a movimentação de cadeiras de rodas; alturas inadequadas do mobiliário como gavetas de armários posicionadas próximo ao piso e estantes com prateleiras muito altas. Portanto, de acordo com Alexandre e Rogante (2000) é necessário observar se o espaço físico está adequado para não restringir os movimentos, sendo que para isso deve-se observar a disposição do mobiliário, examinando o local e removendo obstáculos;

A inadequação da altura das superfícies induz o trabalhador a curvar sua coluna vertebral ao preparar medicamentos, elaborar relatórios ou higienizar/pegar materiais; predispondo o aparecimento de lombalgias e lesões osteomusculares (MARZIALE e CARVALHO, 1998; ROSA et al., 2009).

\section{MOBILIÁRIO E EQUIPAMENTOS}

A existência de cadeiras de rodas e macas com rodas de difícil movimentação compromete a condução desses equipamentos pelos corredores do hospital, exigindo o emprego de um esforço físico maior por parte dos trabalhadores, conforme apontam Rossi; Rocha e Alexandre (2001) e Comélio e Alexandre (2005). Outras observações feitas por Alexandre e Rogante (2000) e Rossi; Rocha e Alexandre (2001) foram a ausência de suporte em cadeiras de rodas e macas obrigando o trabalhador a segurar o frasco de soro elevado, durante o trajeto, enquanto conduz o equipamento e o paciente, arriscando-se a desenvolver lesões musculares e a falta de manutenção dos equipamentos que compromete a segurança do paciente, ocasionando esforço físico e stress à enfermagem. Alexandre e Angerami (1993) apontam que o controle do ambiente e dos equipamentos é fundamental para o bom andamento do trabalho e para a prevenção de doenças profissionais e acidentes de trabalho.

Assim, o principal objetivo de projetar o ambiente de trabalho é a perfeita adaptação dos mobiliários, máquinas e equipamentos ao trabalhador, para reduzir as posturas e movimentos desagradáveis, minimizando as sobrecargas musculares (ROSA et al., 2009), pois os trabalhadores de enfermagem além de manipular pacientes, também transportam e 
movimentam equipamentos e materiais durante suas atividades de trabalho (ALEXANDRE, 1998).

\section{CONSIDERAÇÕES:}

Alexandre e Angerami (1993) apontam que o controle do ambiente e dos equipamentos é fundamental para o bom andamento do trabalho e para a prevenção de doenças profissionais e acidentes de trabalho. As mesmas autoras colocam que a falta de manutenção de equipamentos é um dos fatores que torna o trabalho mais árduo para os trabalhadores de hospital.

Cada unidade do hospital tem problemas ergonômicos comuns a todas e, outros específicos, sendo importante que se projete adequadamente o arranjo físico e dimensões da área de trabalho bem como os equipamentos e mobiliários do local (Alexandre, 1998). Portanto, é de extrema importância a participação da enfermagem no planejamento do ambiente físico hospitalar.

Promover cursos de Educação permanente e treinamento é imprescindível para a melhoria do empenho profissional e da prestação da assistência (ZANON e MARZIALE, 2000; ROSA et al., 2009). Facilidades na mudança de escala ou de setor aos funcionários interessados e existência de pausas para descanso no horário de trabalho, favorecem a segurança e aumentam a motivação e satisfação pelo trabalho (PEREIRA FILHO; OLIVEIRA e CARDOSO, 2006).

A realização de palestras educativas sobre os princípios ergonômicos proporciona aos trabalhadores um maior conhecimento sobre o corpo e seu processo de desgaste, possibilita uma reflexão sobre mudanças de comportamento e desperta a responsabilidade individual para o auto-cuidado (REIMBERG, 2006). Desta forma o profissional passa a ser mais atuante na prevenção dos distúrbios músculo-esqueléticos relacionados ao trabalho.

\section{CONCLUSÃO}

A saúde do trabalhador é essencial para o desenvolvimento de qualquer instituição de saúde. Porém se observa maior cobrança por produtividade e desempenho, sem que sejam oferecidas condições favoráveis para que o profissional possa desenvolver suas funções sem prejuízos à saúde. Na enfermagem, as baixas remunerações, a sobrecarga de trabalho, a rotina estressante e cansativa acabam agravando essa situação. É essencial um planejamento de ações, com participação da enfermagem, com o intuito de melhorar as condições de trabalho; levar a um contentamento da equipe e, consequentemente, preservar a saúde dos trabalhadores, assegurando maior rendimento profissional e qualidade na assistência prestada ao paciente.

\section{REFERÊNCIAS BIBLIOGRÁFICAS}

1. ALEXANDRE, N.M.C. et al. Aspectos ergonômicos e posturais em centro de material. Revista da Escola de Enfermagem da USP, v. 26, n. 1, p. 87- 94, jan. 1992.

2. ALEXANDRE, N.M.C.; ANGERAMI, E.L.S. Avaliação de determinados aspectos ergonômicos no transporte de pacientes. Revista Brasileira de Saúde Ocupacional, v. 21, n. 1, p. 81-90. jan. 1993. 
3. ALEXANDRE, N.M.C.; ANGERAMI, E.L.S.; MOREIRA FILHO, D.C. Dores nas costas e enfermagem. Revista da Escola de Enfermagem da USP, v. 30, n. 2, p. 267-85, ago. 1996.

4. ALEXANDRE, N.M.C. Aspectos ergonômicos relacionados com o ambiente e equipamentos hospitalares. Revista Latino-Americana de Enfermagem, v. 6, n. 4, p. 103-9, out. 1998.

5. ALEXANDRE, N.M.C.; ROGANTE, M.M. Movimentação e transferência de pacientes: aspectos posturais e ergonômicos. Revista da Escola de Enfermagem da USP, v. 34, n. 2, p. 165-73, jun. 2000.

6. BRASIL. Ministério da Saúde. Instrução normativa INSS/DC n ${ }^{\circ} 98$, de 05 de dezembro de 2003. Aprova Norma Técnica sobre lesões por Esforços Repetitivos - LER ou Distúrbios Osteomusculares Relacionados ao Trabalho - DORT. Brasília: Ministério da Saúde; 2003.

7. COMÉLIO, M.E.; ALEXANDRE, N.M.C. Avaliação de uma cadeira de banho utilizada em ambiente hospitalar: uma abordagem ergonômica. Revista Brasileira de Enfermagem, v. 58, n. 4, p. 405-10, jul-ago. 2005.

8. COURY, H.J.C.G.; MOREIRA, R.F.C.; DIAS, N.B. Efetividade do exercício físico em ambiente ocupacional para controle da dor cervical, lombar e do ombro: uma revisão sistemática. Revista Brasileira de Fisioterapia, v. 13, n. 6, p. 461-79, nov.-dez. 2009.

9. DEL VALLE ROYAS, A.; MARZIALE, M.H.P. A situação de trabalho do pessoal de enfermagem no contexto de um hospital argentino: um estudo sob a ótica da ergonomia. Revista Latino-Americana de Enfermagem, v. 9, n. 1, p. 102-8, jan. 2001.

10. GALLASCH, C.H.; ALEXANDRE, N.M.C. Avaliação dos riscos ergonômicos durante a movimentação e transporte de pacientes em diferentes unidades hospitalares. Revista de Enfermagem UERJ, v. 11, n. 3, p. 252-60, set.-dez. 2003.

11. GURGUEIRA, G.P.; ALEXANDRE, N.M.C.; CORRÊA FILHO, H.R. Prevalência de sintomas musculoesqueléticos em trabalhadoras de enfermagem. Revista LatinoAmericana de Enfermagem, v. 11, n. 5, p. 608-13, set.-out. 2003.

12. MAGNAGO, T.S.B.S. et al. Distúrbios musculoesqueléticos em trabalhadores de enfermagem: associação com condições de trabalho. Revista Brasileira de Enfermagem, v. 60, n. 6, p. 701-5, nov.-dez. 2007.

13. MAGNANO, T.S.B.S. et al. Condições de trabalho, características sóciodemográficas e distúrbios musculoesqueléticos em trabalhadores de enfermagem. Acta Paulista de Enfermagem, v. 23, n. 2, p. 187-93, mar.-abr. 2010.

14. MARZIALE, M.H.P.; CARVALHO, E.C. Condições ergonômicas do trabalho da equipe de enfermagem em unidade de internação de cardiologia. Revista LatinoAmericana de Enfermagem, v. 6, n. 1, p. 99-117, jan. 1998.

15. MARZIALE, M.H.P.; ROBAZZI, M.L.C.C. O trabalho de enfermagem e a ergonomia. Revista Latino-Americana de Enfermagem, v. 8, n. 6, p. 124-7, dez. 2000.

16. PEREIRA FILHO, R.L.; OLIVEIRA, M.M.C.; CARDOSO, M.V.L.M.L. Riscos ergonômicos na prática de enfermagem de um centro obstétrico. Revista Rede de Enfermagem do Nordeste, v. 7, n. 1, p. 17-26, jan.-abr. 2006.

17. RADOVANOVIC, C.A.T.; ALEXANDRE, N.M.C. Desenvolvimento de um instrumento para avaliar a movimentação e transferência de clientes: um enfoque ergonômico. Revista da Escola de Enfermagem da USP, v. 36, n. 3, p. 231-9, set. 2002. 
18. REIMBERG, C. Trabalhadores Invisíveis. Revista Proteção, v. 178, n. 1, p. 60-69, jan. 2006.

19. ROSA, L.A.M. et al. Ergonomia: mobiliário adequado não é suficiente para evitar agravos ocupacionais. Revista Proteção, v. 216, n. 1, p. 60-64, jan. 2009.

20. ROSSI, C.G.; ROCHA, R.M.; ALEXANDRE, N.M.C. Aspectos ergonômicos na transferência de pacientes: um estudo realizado com trabalhadores de uma central de transportes de um hospital universitário. Revista da Escola de Enfermagem da USP, v. 35, n. 3, p. 249-56, set. 2001.

21. ZANON, E.; MARZIALE, M.H.P. Avaliação da postura corporal dos trabalhadores de enfermagem na movimentação de pacientes acamados. Revista da Escola de Enfermagem da USP, v. 34, n. 1, p. 26-36, mar. 2000. 